\title{
SIMULATION OF DISK-DISK ENCOUNTERS WITH CO-MOVING POLAR GRIDS
}

\author{
Heikki Salo \\ Observatory, University of Helsinki, Finland
}

\begin{abstract}
Two-grid simulation method combining advantages of both polar and cartesian mesh-codes is described. In addition to stellar component reacting solely to gravitational forces, gas component is included with dissipatively colliding particles. This allows fairly realistic simulation of planar encounters where both systems contain star+gas disks.
\end{abstract}

\section{Introduction}

Nowadays there exists a wealth of data suggesting connection between galaxy-galaxy interactions and the different kinds of star formation and nuclear activity manifested by them. However, this connection is all but simple: for example there seems to be no correlation between the mutual separation of the systems and the level of activity, implying that there must be a large number of factors governing the response to tidal perturbations. These probably include, in addition to the strength and geometry of the external perturbation, for example the internal dynamical state of the systems as well as the amount of gas and its distribution before the encounter. Also, mass transfers between the components might in some cases have significance. Most importantly, even individual galaxy encounter might turn out to be an evolutionary process with a whole time sequence of qualitatively different types of behaviour.

In order to address the connection between encounters and different types of activity, a new $\mathrm{N}$-body code has been constructed, including both stellar and gaseous components of the galaxies. Therefore in addition to previously well-studied tidal phenomena induced in the mass-distributions of the systems, we can also get a handle to the behaviour of gas, and thus to the probable distribution of recent star formation regions, more closely related to the apparant luminosity distributions. The choice of the simulation model is based on the desire to be able to perform fast systematic surveys of as many as possible different model parameters of the systems. Therefore, 2-dimensional polar-mesh approach was chosen, extended to model two disks simultaneously. Here this method is briefly described together with few preliminary examples of disk-disk simulations.

\section{Simulation method}

Two dimensional particle-mesh codes, where total CPU time-consumption scales proportional to particle number, offer a fast method for surveys of the dynamical evolution of essentially flat systems. Self-gravity of the disk is typically calculated in rectangular or polar coordinate grids, by employing FFT-methods. Halos are treated as an inert component, based on the fact that due to their large velocity dispersion they react very weakly on perturbations, at least as long as penetrating encounters are excluded. The advantage 
of using cartesian grids lies in the fact that simple explicit leapfrog method can be used for orbital integrations. Also the application of FFT is very straight forward, as well as extension to 3 -dimensional systems. On the other hand, logarithmic polar grids can offer a much better resolution in the central parts of the system, and are thus very efficient when simulating centrally condensated disks. Therefore, much smaller number of mesh boxes is needed to cover the area of interest with necessary resolution. However, there are disadvantages in using polar coordinates: implicit leapfrog schemas are needed as well as special treatment at the center of the grid. Also, if both systems are to possess a full disk, simple polar methods are not suitable, and cartesian grids covering both systems are usually applied. Since a large area must then be covered with equally sized bins, resolution is inevitably rather poor.

Present simulations try to combine the advantages of both cartesian and polar grids. The gravitational potential is evaluated simultaneously in two moving, mutually overlapping polar grids whose centers are attached to centers of the two halos (Fig. 1). For each particle coordinates are calculated with respect to both grids, while its mass is assigned only to the disk it originally belonged. At each step the logarithmic polar grid method of Miller (1976) is used for both grids separately after which forces from both grids are added together. Thus the good resolution is retained near the centers of both systems. On the other hand, orbital calculations, both for the particles and the halo centers, are performed in cartesian coordinate system, thus allowing the use of fast explicit leapfrog. This fact more than outweigths the extra time consumption in transformations between coordinate systems. Notice, that allowing the halos to move freely in the inertial frame is totally equivalent to the usual method of fixing the coordinate system to the main galaxy and letting the point-mass perturber to exert both direct and indirect forces. Since logaritmic grids can cover large areas with very limited number of mesh points there is practically no

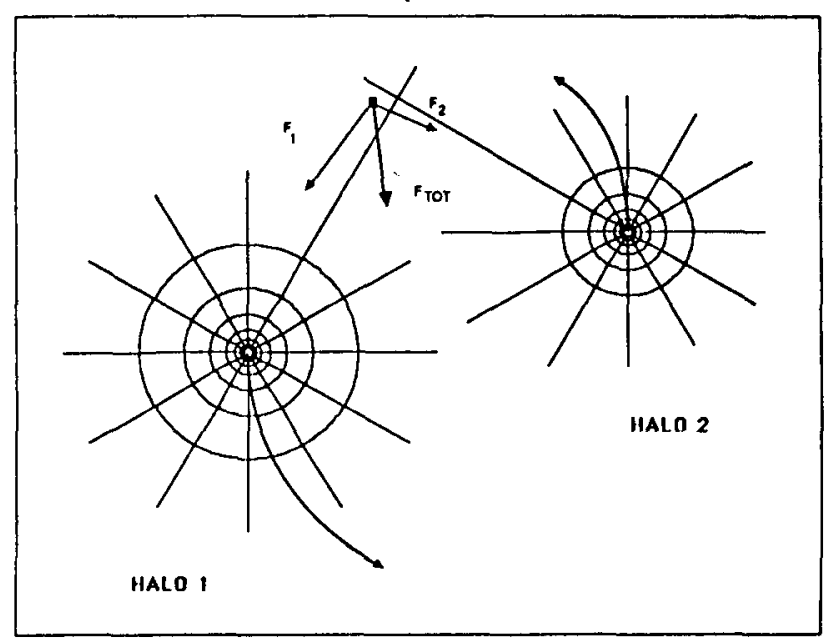

Figure 1. Schematic representation of the 2-grid coordinate system. Actual runs employ 36 azimuthal and 48 radial bins covering the area from 0.005 to about 20 initial disk radii. 
restrictions on the distance between the systems: large initial separations can be used, thus assuring that systems have time to settle into steady state before tidal forces start to influence their dynamics.

\section{Gas component}

A certain fraction of the particles represents gas clouds, which are allowed to experience dissipative impacts. In contrast to typical treatment in $\mathrm{N}$-body simulations where collisions are assumed to take place whenever two clouds fall into same 'collisional bin', here the orbits are followed to the exact location of impact, as is commonly done in collisional simulations of planetary rings (e.g. Salo, 1987, 1990a; Wisdom and Tremaine, 1988). Due to the simplicity of the leapfrog algorithm, even multiple collisions/time-step/cloud can be correctly calculated with iterative methods. Compared to bin methods, exact collisional calculations make it possible to follow to greater accuracy the formation of shock fronts in gas due to tidal perturbation. This is especially important in the case the gas disks penetrate each other, since then due to large streaming motions many impacts would be lost in collision bin methods (see legend for Fig. 3) and the results of the experiment might be even qualitatively different.

\section{Applications and future refinements}

So far the code has been applied to study tidal triggering of bars (Salo, 1990b), which has previously been shown (Noguchi, 1987, 1988) to be potentially able to cause large-scale infall of gas to central regions of galaxies, thus offering a possible source of fresh material for active nuclei. These experiments confirmed Noguchi's results, but also illustrated the dependence of bar formation on several factors like rotation curve, disk/halo mass-ratio, stressing the point that gas infall not necessarily occur in interaction, especially not for systems with high degree of central condensation. Here a few illustrative examples are shown for disk-disk encounters: Fig. 2 displays the different evolutionary phases during an encounter (including formation of shock fronts in various locations, as well as the final bar driven infall) while Fig. 3 gives an example how internal kinematics (sense of rotation) can affect induced activity. In future the code will be used for systematic surveys of activity in disk-disk encounters, and more realistic treatments for the cloud ensemble will be explored, including models where accretional and fragmentational evolution of clouds is taken into account. Possibility to extend multiple polar-grids to 3 dimensions is also investigated.

\section{References}

Miller, R.: 1976, J. Comp. Phys. 21, 400.

Noguchi, M.: 1987, M.N.R.a.S. 228, 635.

Noguchi, M.: 1988, Astron. Astrophys. 203, 259.

Salo, H.: 1987, Icarus 70, 37.

Salo, H.: 1990a, submitted to Icarus.

Salo, H.: 1990b, submitted to Astron. Astrophys.

Wisdom, J. and Tremaine, S.: 1988, A.J. 95, 925 


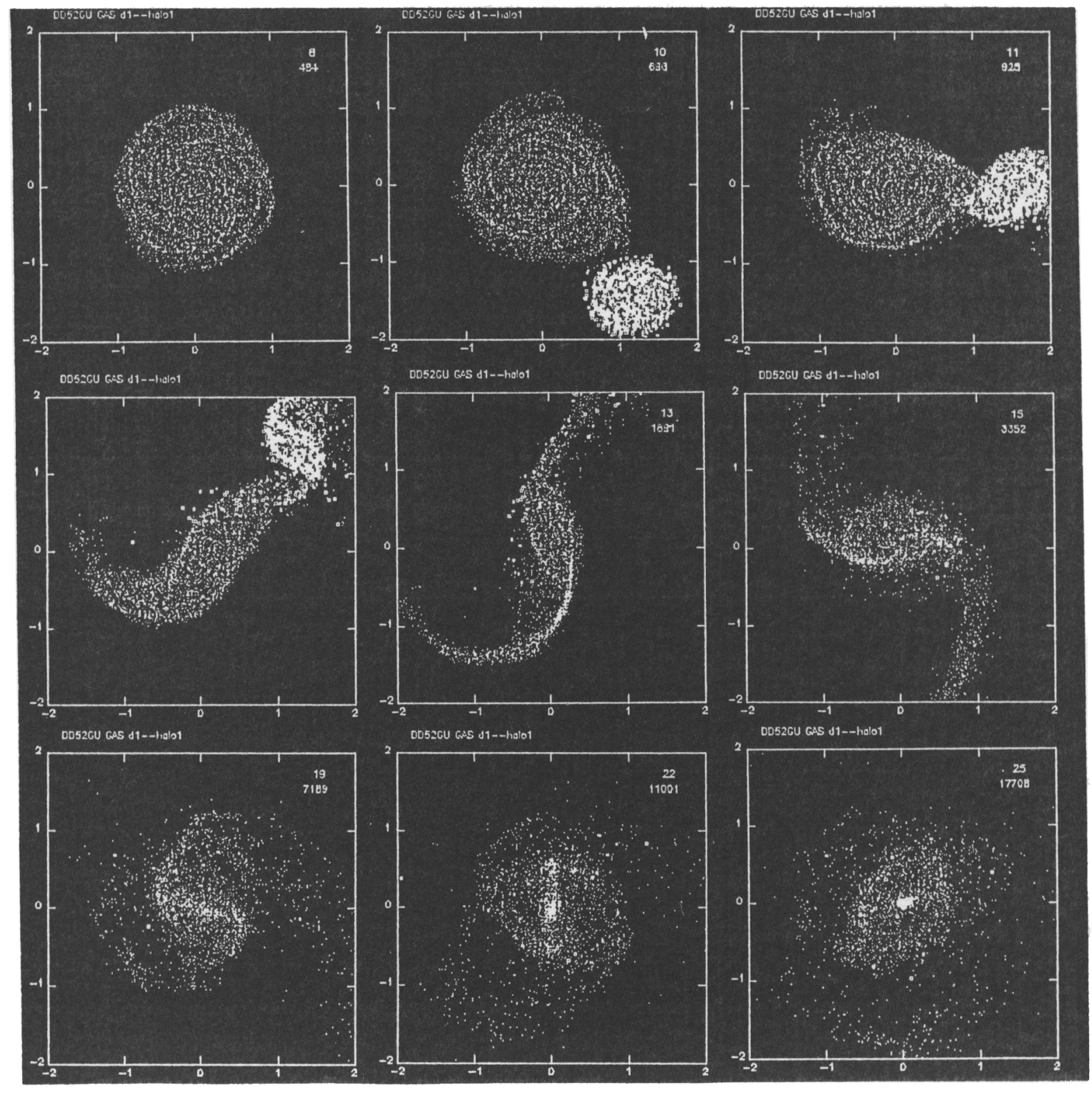

Figure 2. Example of disk-disk encounter in the frame centered at the main galaxy. Stellar components were represented with $20000+10000$ particles (Toomre disks) containing 1/3 of the total mass, the rest being in inert halos (Plummer spheres). Companion galaxy $\left(m_{2}=0.5 m_{1}, r_{2}=0.5 r_{1}\right)$ was in direct parabolic orbit with minimum separation of $r_{1}+r_{2}$. Scale-radii for both the disk and the halo distributions were 0.5 and 0.2 times the initial disk radii for the main galaxy and the companion, respectively. Gas components (shown) were represented by $4000+1000$ uniformly distributed clouds. One can see the formation of weak spiral structures before the encounter, as well as the shocks caused by direct tidal perturbations. In post-encounter phase stellar bar is formed (isolated model is stable against bar formation), forcing gas alignment as well as flow into nuclear regions due to angular momentum exchange in non-axisymmetric bar-potential. In the more compact secondary galaxy no infall occurred but a gas ring was formed. Notice that frames are not evenly spaced in time. This simulation with 3000 steps required about 2000 secs in Cray $\mathrm{X}$-MP (only about 300 secs without gas cloud collisions). Frames are extracted from a MacIntosh Hypercard-animation sequence, able to display about $10 \mathrm{frames} / \mathrm{sec}$ in MacII cx (stack developed by T. Tokkonen at the Dept. of Astronomy, Univ. of Oulu, Finland). 

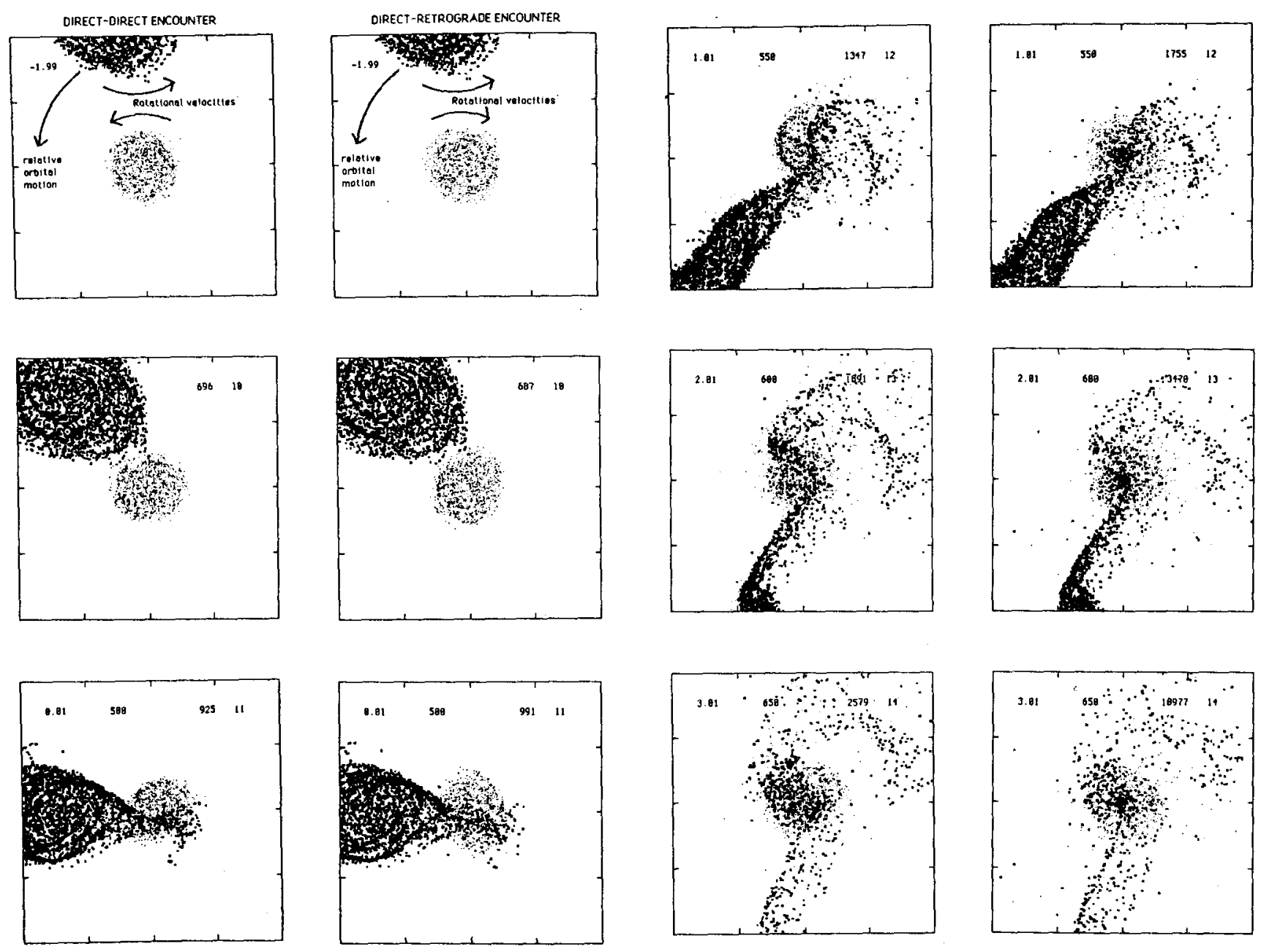

Figure 3. Details of the behaviour of the companion galaxy in the simulation of Fig.2. (direct-direct) and in another run with same parameters except that the direction of the rotation of the companion was reversed (direct-retrograde). In the direct-direct case the response of stellar components is strongest (both develop bridges etc.) but due to superposition of orbital and rotational velocities gas reacts strongest in the retrograde case where large net streaming motions occur between clouds originating from different systems. This leads to strong shocks and rapid loss of rotational angular momentum, and fast infall of gas just past the closest encounter, when the tidal bridge from the main galaxy hits the secondary. On the direct-direct case no infall occurred in the secondary. In an additional direct-retrograde experiment using collision bin method, the infall was considerably delayd, by almost 5 galaxy rotations. Notice, however, that the role of mass transfer is here considerably exaggareted due to planar geometry. 